Short Communication

\title{
Human population genetic structure detected by pain-related mu opioid receptor gene polymorphisms
}

\author{
Eduardo Javier López Soto ${ }^{1,2}$ and Cecilia Inés Catanesi ${ }^{1,3}$ \\ ${ }^{1}$ Laboratory of Genetic Diversity, Multidisciplinary Institute of Cell Biology, Consejo Nacional de \\ Investigaciones Cientificas y Técnicas, La Plata, Buenos Aires, Argentina. \\ ${ }^{2}$ Laboratory of Electrophysiology, Multidisciplinary Institute of Cell Multidisciplinary Institute of Cell \\ Biology, Consejo Nacional de Investigaciones Científicas y Técnicas, La Plata, Buenos Aires, Argentina. \\ ${ }^{3}$ Facultad de Cs. Naturales y Museo UNLP, La Plata, Buenos Aires, Argentina.
}

\begin{abstract}
Several single nucleotide polymorphisms (SNPs) in the Mu Opioid Receptor gene (OPRM1) have been identified and associated with a wide variety of clinical phenotypes related both to pain sensitivity and analgesic requirements. The A118G and other potentially functional OPRM1 SNPs show significant differences in their allele distributions among populations. However, they have not been properly addressed in a population genetic analysis. Population stratification could lead to erroneous conclusions when they are not taken into account in association studies. The aim of our study was to analyze OPRM1 SNP variability by comparing population samples of the International Hap Map database and to analyze a new population sample from the city of Corrientes, Argentina. The results confirm that OPRM1 SNP variability differs among human populations and displays a clear ancestry genetic structure, with three population clusters: Africa, Asia, and Europe-America.
\end{abstract}

Keywords: OPRM1, SNPs, A118G, AMOVA, population genetics.

Received: October 10, 2014; Accepted: February 24, 2015.

The human OPRM1 gene encodes the mu opioid receptor (MOR), a G-protein coupled receptor associated with a wide number of complex neurobiological traits related to pain. Several single nucleotide polymorphisms (SNPs) in OPRM1 have been extensively associated with pain sensitivity, tolerance, and analgesic response (Fernandez Robles et al., 2012). Therefore, the study of OPRM1 genetic variations is clinically relevant.

OPRM1 SNPs can modify receptor properties. The most studied SNP, A118G (rs1799971), is a frequent nonconservative substitution in exon I, which gives rise to an amino acid exchange from asparagine to aspartate at position 40 of the protein (N40D) (Bergen et al., 1997). Significant differences in the agonist affinity and potency in MOR activity have been reported for N40D (Bond et al., 1998; Lopez Soto and Raingo 2012). Additionally, several studies have correlated N40D with higher pain score and analgesic requirements in clinical studies (Campa et al., 2008; Sia et al., 2008). In contrast, other studies failed to detect

Send correspondence to Eduardo J López Soto. Molecular Genetics Laboratory and Electrophysiology Laboratory, Multidisciplinary Institute of Cell Biology, Consejo Nacional de Investigaciones Científicas y Técnicas, Calle 526 y Camino Belgrano e/10 y $11 \mathrm{~S} / \mathrm{N}$, La Plata 1900, Buenos Aires, Argentina. E-mail: ejlopezsoto@gmail.com, jlopezsoto@ imbice.gov.ar. these clinical associations or presented opposite results (Lopez Soto et al., 2013). Therefore, further research is required to elucidate the physiological impact of A118G.

Other SNPs, like C17T (rs1799972), T1443A (rs540825) and C440G (rs17174794), could also change the amino acid sequence of MOR (A6V, H402Q, and S147C, respectively). Additionally, some SNPs located in intronic regions, like IVS2+691C/G (rs2075572), or producing synonymous changes in exonic regions, like C1570T (rs562859), may not modify the MOR amino acidic sequence, but have been associated with pain sensitivity and postulated to be a risk factor for schizophrenia and heroin abuse (Shabalina et al., 2009; Ding et al., 2013; Sillivan et al., 2013). Thus, coding and non-coding OPRM1 SNPs could modify MOR properties and physiology.

Numerous association studies have reported significant differences in OPRM1 SNP allele distributions among populations (Bergen et al., 1997; Bond et al., 1998; Gelernter et al., 1999; Tan et al., 2009) but this has not been addressed in a genetic population analysis. It is well established that A118G can reach a specific range of allele frequency depending on the population ancestry under study: Europeans 10-30\%; Asians 36-50\%; Hispanics 15-18\% and Africans 1-17\% (Bergen et al., 1997; Bond et al., 1998; 
Gelernter et al., 1999; Tan et al., 2009). Our previous results confirm that A118G SNP genetic variance analysis is able to detect a population genetic structure (Lopez Soto et al., 2013). In contrast, rs540825 and rs562859 polymorphisms are located in an alternative exon, defining the MOR-1X isoform of the OPRM1 gene in the intracellular domain of the receptor (Smith et al., 2005). They are separated by 128 base pairs and linkage disequilibrium between these two SNPs is moderate $(\mathrm{r} 2=0.51)$ (Garriock et al., 2010). Interestingly, only the rs 540825 genetic frequencies differ by a large margin between Afro-Americans and European-Americans (Smith et al., 2005). Both population structure and recent admixture events could be a problem in association studies, leading to false positive or false negative associations and consequently erroneous conclusions (Ziv and Burchard 2003; Freedman et al., 2004). Hence, the aim of the study was to analyze OPRM1 SNP variability and to investigate the genetic structure of population samples from the International HapMap database and a mixed population sample from Argentina in relation to gene frequency distribution of several pain-related OPRM1 SNPs.

OPRM1 variability has been widely analyzed in worldwide populations (Levran et al., 2011), but the frequencies of such polymorphisms in Argentinian people have not been established yet. Considering that there is an important European and Native American genetic contribution (Diaz-Lacava et al., 2011; Avena et al., 2012), these components might impact on the frequencies of OPRM1 SNPs.

The current analysis included a population sample of 108 non-related donors from the city of Corrientes, (province of Corrientes, Argentina, CTES), collected under medical supervision in public hospitals. Each individual was requested to voluntarily participate in this study, and was asked to sign an Informed Consent. This study was approved by the Ethics Committee for Biomedical Research from the Multidisciplinary Institute of Cell Biology (IMBICE), La Plata, Argentina.

Six SNPs (rs1799971, rs1799972, rs17174794, rs2075572, rs540825 and rs562859), selected for their ability to change protein function and/or for their population frequencies, were genotyped as previously described (Bergen et al., 1997; Gelernter et al., 1999; Smith et al., 2005). Rs1799971 genotype information from CTES population previously published by our group (Lopez Soto et al., 2013) was included in this analysis and extended to 108 samples. For each marker, the amplified PCR fragments of 6-10 random samples were sequenced as a quality control to confirm the accuracy of the PCR-RFLP results.

For statistical analysis, allele and genotype frequencies of CTES sample were calculated by direct counting. Hardy-Weinberg equilibrium was tested (Exact test, $\mathrm{p}<0.05$ ), genetic distances (Fst) among populations were estimated (Exact test, $\mathrm{p}<0.05$ ), and different genetic structures among populations were tested by Analysis of Molec- ular Variance (AMOVA, permutation, $\mathrm{p}<0.05$ ) using Arlequin v3.5 (Excoffier et al., 2005). In order to detect population structure, nine HapMap populations were included (www.hapmap.org): Utah residents with Northern and Western European ancestry from the CEPH collection (CEU); Han Chinese in Beijing, China (CHB); Chinese in Metropolitan Denver, Colorado (CHD); Gujarati Indians in Houston, Texas (GIH); Japanese in Tokyo, Japan (JPT); Mexican ancestry in Los Angeles, California (MEX); Maasai in Kinyawa, Kenya (MKK); Tuscan in Italy (TSI) and African ancestry in Southwest USA (ASW). Population samples from Luhya in Webuye, Kenya, and Yoruba in Ibadan, Nigeria, were not included because the selected SNPs were genotyped in less than $50 \%$ of the total samples available.

All SNPs analyzed in the CTES sample were biallelic and their frequency distribution presented non-significant deviation from Hardy-Weinberg equilibrium (Exact test, $p>0.05$ ). Additionally, it was found that the most frequent variant was the ancestral allele for all the analyzed SNPs, ranging from 0.99 (rs1799972) to 0.53 (rs2075572). On the other hand, while four of the six SNPs (rs1799971, rs2075572, rs540825, rs562859) showed minor allele frequencies exceeding $1 \%$, thus being able to be considered polymorphisms, the other two SNPs (rs1799972 and rs 17174794 ) reached less than $1 \%$ allele frequency so they could be considered as recurrent mutations in CTES sample.

Corrientes is the capital city of the Province of Corrientes, located on the northeast of Argentina. Its population has two main genetic contributions, from Native American and European people. According to autosomal and uniparental markers, the genetic background of the Corrientes population has the same global genetic structure as that of Argentina as a whole (Diaz-Lacava et al., 2011; Avena et al., 2012). In Corrientes, European genetic heritage is mainly autosomal, whereas the mitochondrial gene pool is mostly of Native American ancestry (African heritage is small in all genetic systems). Population substructure based on autosomal information is very small in Argentina (Corach et al., 2010). Therefore, Corrientes might be an adequate population sample to reduce sampling efforts in Argentina in order to perform population genetic studies at the continental level. Thus the CTES sample was included in the analysis of population genetic structure.

The International HapMap Project is the major international research resource to construct a human SNP database that offers information about patterns of variation in specific populations (International HapMap Consortium 2003). Taking advantage of the availability of the HapMap Project SNP database, the six SNPs genotype frequencies of nine populations: CEU, CHB, CHD, GIH, JPT, MEX, MKK, TSI and ASW were included in the OPRM1 genetic variability analysis. Thus, in order to identify geographic structure among these worldwide populations including 
Table 1 - Genetic distances between populations. Values indicate population pairwise Fst. Shadings indicate the continental origin of populations as follows: light gray block = European and American, gray block = Asian, dark gray block = African populations. Asterisks mark statistically significant values (significance test: 1023 permutations, $\mathrm{p}<0.01$ ).

\begin{tabular}{|c|c|c|c|c|c|c|c|c|c|c|}
\hline & CTES & CEU & TSI & MEX & $\mathrm{CHB}$ & CHD & JPT & GIH & ASW & MKK \\
\hline CTES & 0.00000 & & & & & & & & & \\
\hline CEU & -0.03060 & 0.00000 & & & & & & & & \\
\hline TSI & -0.00256 & -0.00984 & 0.00000 & & & & & & & \\
\hline MEX & -0.00880 & -0.02114 & -0.00003 & 0.00000 & & & & & & \\
\hline $\mathrm{CHB}$ & $0.07178 *$ & $0.14913 *$ & $0.06052 *$ & $0.07697 *$ & 0.00000 & & & & & \\
\hline CHD & $0.11305^{*}$ & $0.17014 *$ & $0.10448 *$ & $0.11007 *$ & -0.00212 & 0.00000 & & & & \\
\hline JPT & $0.14357 *$ & $0.20374 *$ & $0.13548 *$ & $0.14233 *$ & 0.00785 & -0.00043 & 0.00000 & & & \\
\hline $\mathrm{GIH}$ & $0.07846^{*}$ & $0.10898 *$ & $0.07556^{*}$ & $0.07294 *$ & -0.01576 & -0.00338 & 0.00110 & 0.00000 & & \\
\hline ASW & $0.04789 *$ & $0.05385^{*}$ & $0.03497 *$ & $0.04293 *$ & $0.17912 *$ & $0.21191 *$ & $0.26375^{*}$ & $0.17033 *$ & 0.00000 & \\
\hline MKK & $0.03454 *$ & $0.02272 *$ & $0.02544 *$ & $0.03417^{*}$ & $0.16738^{*}$ & $0.21235^{*}$ & $0.25623 *$ & $0.17665^{*}$ & -0.00014 & 0.00000 \\
\hline
\end{tabular}

CTES, pairwise variance of population gene frequencies was calculated (Fst) (Excoffier et al., 2005) (Table 1). Statistical differences were found $(\mathrm{p}<0.01)$ among populations tracing ancestry clusters, except between American (CTES and MEX) and European (CEU and TSI) populations, which were indistinguishable by the Fst index. This approach allowed to recognize three clusters: one composed of American and European populations (CTES, CEU, TSI and MEX), one composed of Asian populations (CHB, CHD, JPT and GIH) and another one composed of African populations (ASW and MKK).

Aditionally, two different grouping criteria were considered in order to test genetic structures based on OPRM1 SNPs variability by AMOVA (Table 2): "all populations" criteria, which groups all populations together in one group; and "three groups" criteria, which includes the three ancestry clusters: the European and American group (EuAm), the Asian group (As), and the African group (Af). The results showed that when one group was defined ("all populations"), variation among populations represented $8.9 \%$ of the total variation (Table 2), thus indicating that the genetic variation is ancestrally structured. Additionally, when considering the ancestry (EuAm, As and Af) it was found that the variance component among groups reached a significant $12.81 \%$, with no differences among populations within groups. Thus, a genetic structure based on ancestry, consisting of three groups: the European-American, the Asian, and the African group was confirmed.

On the other hand, whereas Fst analysis found no differentiation between American and European populations, the possibility of identifying any genetic structure among these populations was also tested. AMOVA was used by two criteria: "European ancestry populations" which includes CTES, CEU, TSI and MEX in a single group, and "two groups" which corresponds to American (Am) and European $(\mathrm{Eu})$ populations in separated groups. AMOVA showed no significant variation among populations, or between the two groups in either case. Hence, identifying a genetic structure in American and European populations using OPRM1 SNP variability by Fst and AMOVA was not possible.

By means of two population genetic approaches, Fst and AMOVA, it was found that all the analyzed population samples were grouped into three clusters corresponding to different human ancestral lineages: African, Asian, and European-American. Several studies have demonstrated that since the time of colonization, the genetic flow between America and Europe has been very important and has had a dramatic impact on the current genetic background of North, Central, and South American populations (Diaz-

Table 2 - Analysis of Molecular Variance (AMOVA) among worldwide populations. Values indicate variance component in hierarchical levels in four genetic structures tested. Asterisks mark statistically significant values (significance test: 1023 permutations, $\mathrm{p}<0.05$ ).

\begin{tabular}{lccc}
\hline Pairwise differences & \multicolumn{3}{c}{$\%$ variation } \\
\cline { 2 - 4 } & Within populations & Among populations (within groups) & Among groups \\
\hline All populations & $91.11^{*}$ & $8.89^{*}$ & - \\
3 groups (EuAm / As / Af) & $87.78^{*}$ & -0.58 & $12.81^{*}$ \\
European ancestry populations & 101.29 & -1.29 & - \\
2 groups $(\mathrm{Eu} / \mathrm{Am})$ & 102.47 & -0.94 & -0.53 \\
\hline
\end{tabular}

$\mathrm{EuAm}=$ European and American populations, As $=$ Asian populations, $\mathrm{Af}=\mathrm{African}$ populations, $\mathrm{Eu}=$ European populations Am $=\mathrm{American}$ populations. 
Lacava et al., 2011; Avena et al., 2012). Thus, the historical demographic events could explain the similarity of American and European populations in OPRM1 SNP variability. The main limitation of our study is the small number of SNPs included to perform the genetic population analysis leading to a possible loss of sensitivity in detecting a population structure and an over- or underestimation of group differentiation. Despite this, our results should be considered in OPRM1 association studies, for it has been suggested that population stratification between controls and cases due to genetic background might cause false associations between a SNP and a specific phenotype.

In conclusion, the study consistently found that OPRM1 SNP variability clearly displays a population structure by ancestry. Additionally, this study expanded our previous results (Lopez Soto et al., 2013) and it is a contribution to coding regions polymorphism knowledge in Argentinian population. In the future, this information might be of potential application in palliative therapies, since OPRM1 polymorphisms affect a wide range of responses in pain perception pathway.

\section{Acknowledgments}

EJLP and CIC were supported by CONICET and PICT2011-0626 grants.EJLP is a postdoc of CONICET, and $\mathrm{CIC}$ is a researcher of CONICET.

\section{References}

Avena S, Via M, Ziv E, Perez-Stable EJ, Gignoux CR, Dejean C, Huntsman S, Torres-Mejia G, Dutil J, Matta JL et al. (2012) Heterogeneity in genetic admixture across different regions of Argentina. PLoS One 7:e34695-34704.

Bergen AW, Kokoszka J, Peterson R, Long JC, Virkkunen M, Linnoila M and Goldman D (1997) Mu opioid receptor gene variants: Lack of association with alcohol dependence. Mol Psychiatry 2:490-494.

Bond C, LaForge KS, Tian M, Melia D, Zhang S, Borg L, Gong J, Schluger J, Strong JA, Leal SM et al. (1998) Single-nucleotide polymorphism in the human mu opioid receptor gene alters beta-endorphin binding and activity: Possible implications for opiate addiction. Proc Natl Acad Sci USA 95:9608-9613.

Campa D, Gioia A, Tomei A, Poli P and Barale R (2008) Association of $\mathrm{ABCB} 1 / \mathrm{MDR} 1$ and OPRM1 gene polymorphisms with morphine pain relief. Clin Pharmacol Ther 83:559-566.

Corach D, Lao O, Bobillo C, van Der Gaag K, Zuniga S, Vermeulen M, van Duijn K, Goedbloed M, Vallone PM, Parson $\mathrm{W}$ et al. (2010) Inferring continental ancestry of argentineans from Autosomal, Y-chromosomal and mitochondrial DNA. Ann Hum Genet 74:65-76.

Diaz-Lacava A, Walier M, Penacino G, Wienker TF and Baur MP (2011) Spatial assessment of Argentinean genetic admixture with geographical information systems. Forensic Sci Int Genet 5:297-302.

Ding S, Chen B, Zheng Y, Lu Q, Liu L and Zhuge QC (2013) Association study of OPRM1 polymorphisms with Schizophrenia in Han Chinese population. BMC Psychiatry 13:107.
Excoffier L, Laval G and Schneider S (2005) Arlequin (version 3.0): An integrated software package for population genetics data analysis. Evol Bioinform Online 1:47-50.

Fernandez Robles CR, Degnan M and Candiotti KA (2012) Pain and genetics. Curr Opin Anaesthesiol 25:444-449.

Freedman ML, Reich D, Penney KL, McDonald GJ, Mignault AA, Patterson N, Gabriel SB, Topol EJ, Smoller JW, Pato $\mathrm{CN}$ et al. (2004) Assessing the impact of population stratification on genetic association studies. Nat Genet 36:388-393.

Garriock HA, Tanowitz M, Kraft JB, Dang VC, Peters EJ, Jenkins GD, Reinalda MS, McGrath PJ, von Zastrow M, Slager SL et al. (2010) Association of mu-opioid receptor variants and response to citalopram treatment in major depressive disorder. Am J Psychiatry 167:565-573.

Gelernter J, Kranzler H and Cubells J (1999) Genetics of two mu opioid receptor gene (OPRM1) exon I polymorphisms: Population studies, and allele frequencies in alcohol- and drugdependent subjects. Mol Psychiatry 4:476-483.

Levran O, Awolesi O, Linzy S, Adelson M and Kreek MJ (2011) Haplotype block structure of the genomic region of the mu opioid receptor gene. J Hum Genet 56:147-155.

Lopez Soto EJ and Raingo J (2012) A118G Mu Opioid Receptor polymorphism increases inhibitory effects on $\mathrm{CaV} 2.2$ channels. Neurosci Lett 523:190-194.

Lopez Soto EJ, Agosti F, Catanesi CI and Raingo J (2013) Impact of A118G Polymorphism on the Mu Opioid Receptor Function in Pain. Journal of Pain \& Relief 02:2-6.

Shabalina SA, Zaykin DV, Gris P, Ogurtsov AY, Gauthier J, Shibata K, Tchivileva IE, Belfer I, Mishra B, Kiselycznyk C et al. (2009) Expansion of the human mu-opioid receptor gene architecture: Novel functional variants. Hum Mol Genet 18:1037-1051.

Sia AT, Lim Y, Lim EC, Goh RW, Law HY, Landau R, Teo YY and Tan EC (2008) A118G single nucleotide polymorphism of human mu-opioid receptor gene influences pain perception and patient-controlled intravenous morphine consumption after intrathecal morphine for postcesarean analgesia. Anesthesiology 109:520-526.

Sillivan SE, Whittard JD, Jacobs MM, Ren Y, Mazloom AR, Caputi FF, Horvath M, Keller E, Ma'ayan A, Pan YX et al. (2013) ELK1 transcription factor linked to dysregulated striatal mu opioid receptor signaling network and OPRM1 polymorphism in human heroin abusers. Biol Psychiatry 74:511-519.

Smith RJ, Doyle GA, Han AM, Crowley JJ, Oslin DW, Patkar AA, Mannelli P, Demaria PA, Jr., O'Brien C P and Berrettini WH (2005) Novel exonic mu-opioid receptor gene (OPRM1) polymorphisms not associated with opioid dependence. Am J Med Genet B Neuropsychiatr Genet 133B:105-109.

Tan EC, Lim EC, Teo YY, Lim Y, Law HY and Sia AT (2009) Ethnicity and OPRM variant independently predict pain perception and patient-controlled analgesia usage for post-operative pain. Mol Pain 5:32-40.

Ziv E and Burchard EG (2003) Human population structure and genetic association studies. Pharmacogenomics 4:431-441.

Associate Editor: Mara H. Hutz

License information: This is an open-access article distributed under the terms of the Creative Commons Attribution License, which permits unrestricted use, distribution, and reproduction in any medium, provided the original work is properly cited. 